\title{
HALL SUBGROUPS AND STABLE BRAUER CHARACTERS
}

\author{
GABRIEL NAVARRO \\ Departament d'Àlgebra, Facultat de Matemàtiques, Universitat de València, \\ 46100 Burjassot, València, Spain (gabriel@uv.es)
}

(Received 11 October 1999)

\begin{abstract}
Let $H$ be a Hall $\pi$-subgroup of a finite $\pi$-separable group $G$, and let $\alpha$ be an irreducible Brauer character of $H$. If $\alpha(x)=\alpha(y)$ whenever $x, y \in H$ are $p$-regular and $G$-conjugate, then $\alpha$ extends to a Brauer character of $G$.
\end{abstract}

Keywords: Brauer characters; Hall subgroups

AMS 2000 Mathematics subject classification: Primary 20C15; 20C20

\section{Introduction}

Let $G$ be a finite group and let $p$ be a prime number. Suppose that $U \subseteq G$ and let $\alpha \in$ $\operatorname{IBr}(U)$ be an irreducible Brauer character of $U$. We say that $\alpha$ is $G$-stable if $\alpha(x)=\alpha(y)$ whenever $x, y \in U$ are $p$-regular and $G$-conjugate.

We prove the following theorem.

Theorem A. Suppose that $G$ is $\pi$-separable and let $H$ be a Hall $\pi$-subgroup of $G$. If $\alpha \in \operatorname{IBr}(H)$ is $G$-stable, then $\alpha$ extends to some Brauer character of $G$.

If $H \triangleleft G$, then Theorem A is Gallagher's theorem (for ordinary characters) and Dade's theorem (for Brauer characters; see [1] and Theorem (8.13) of [4]). If $p$ does not divide $|H|$, Theorem A was obtained by Isaacs in [2] (with a different approach). As is well known, extendability of characters and control of fusion are closely related.

Corollary B. Suppose that $G$ is $\pi$-separable and let $H$ be a Hall $\pi$-subgroup of $G$. Then every irreducible Brauer character of $H$ extends to some Brauer character of $G$ if and only if whenever $x, y \in H$ are $p$-regular and $G$-conjugate, then $x$ and $y$ are $H$-conjugate.

\section{Proofs}

If $U \subseteq G$, we denote by $U^{0}$ the set of $p$-regular elements of $U$. Also, $\operatorname{cf}\left(U^{0}\right)$ is the complex space of class functions of $U$ defined on $U^{0}$. We say that $\alpha \in \operatorname{cf}\left(U^{0}\right)$ is $G$-stable if $\alpha(x)=\alpha(y)$ whenever $x, y \in U$ are $p$-regular and $G$-conjugate. 
Lemma 2.1. Let $\alpha \in \operatorname{cf}\left(U^{0}\right)$. Then $\alpha$ is $G$-stable if and only if there exists $\xi \in \operatorname{cf}\left(G^{0}\right)$ such that $\xi_{U}=\alpha$.

Proof. Assume that $\alpha$ is $G$-stable. We define some $\xi \in \operatorname{cf}\left(G^{0}\right)$. We put $\xi(x)=0$, say, for every $p$-regular element $x \in G$ lying in no $G$-conjugate of $U$. If, on the other hand, $\operatorname{cl}(x) \cap U$ is non-empty, we set $\xi(x)=\alpha(u)$, where $u$ is any element of $\operatorname{cl}(x) \cap U$. Since $\alpha$ is $G$-stable, it follows that $\xi$ is a well-defined function on $G^{0}$ extending $\alpha$. For the converse, it is clear that if $\alpha$ extends to some $\xi \in \operatorname{cf}\left(G^{0}\right)$, then $\alpha$ is $G$-stable.

Lemma 2.2. Let $\alpha \in \operatorname{cf}\left(U^{0}\right)$. Then $\alpha$ is $G$-stable if and only if

$$
\left(\alpha^{G}\right)_{U}=\alpha\left(\left(1_{U}\right)^{G}\right)_{U} .
$$

Proof. Assume that $\alpha$ is $G$-stable. By Lemma 2.1, let $\xi \in \operatorname{cf}\left(G^{0}\right)$ be such that $\xi_{U}=\alpha$. Now,

$$
\left(\alpha^{G}\right)_{U}=\left(\left(\xi_{U}\right)^{G}\right)_{U}=\left(\xi\left(1_{U}\right)^{G}\right)_{U}=\xi_{U}\left(\left(1_{U}\right)^{G}\right)_{U}=\alpha\left(\left(1_{U}\right)^{G}\right)_{U}
$$

as desired. Assume now that

$$
\left(\alpha^{G}\right)_{U}=\alpha\left(\left(1_{U}\right)^{G}\right)_{U}
$$

and note that $\left(\left(1_{U}\right)^{G}\right)_{U}$ is a $G$-stable class function of $U$ which is never zero. Then

$$
\alpha=\frac{\left(\alpha^{G}\right)_{U}}{\left(\left(1_{U}\right)^{G}\right)_{U}}
$$

is the quotient of two $G$-stable class functions. Hence $\alpha$ is $G$-stable.

From now on, we fix a maximal ideal of the ring of the algebraic integers $\boldsymbol{R}$ containing $p \boldsymbol{R}$, so that for every finite group $G$ we have a uniquely defined set of irreducible Brauer characters $\operatorname{IBr}(G)$ of $G$. We follow the notation of $[4]$.

Let $N \triangleleft G$ and let $\theta \in \operatorname{IBr}(N)$. We denote by $\operatorname{cf}\left(G^{0} \mid \theta\right)$ the complex linear combinations of $\operatorname{IBr}(G \mid \theta)$.

Lemma 2.3. Suppose that $N \subseteq U \subseteq G$ where $N \triangleleft G$. Let $\theta \in \operatorname{IBr}(N)$ and suppose that $\alpha \in \operatorname{IBr}(U \mid \theta)$ is $G$-stable. Then there exists $\phi \in \operatorname{cf}\left(G^{0} \mid \theta\right)$ extending $\alpha$. Also, $T U=G$, where $T$ is the stabilizer of $\theta$ in $G$.

Proof. By Lemma 2.1, let $\hat{\alpha} \in \operatorname{cf}\left(G^{0}\right)$ be an extension of $\alpha$. Write

$$
\operatorname{cf}\left(G^{0}\right)=\operatorname{cf}\left(G^{0} \mid \theta\right) \oplus \Delta,
$$

where $\Delta$ is the $C$-span of those irreducible Brauer characters $\mu$ of $G$ that do not lie over $\theta$. We write $\hat{\alpha}=\phi+\psi$, where $\phi \in \operatorname{cf}\left(G^{0} \mid \theta\right)$ and $\psi \in \Delta$. We claim that $\alpha^{G} \in \operatorname{cf}\left(G^{0} \mid \theta\right)$. By Lemma 2.2, we have that

$$
\left(\alpha^{G}\right)_{U}=\alpha\left(\left(1_{U}\right)^{G}\right)_{U}
$$

Then

$$
\left(\alpha^{G}\right)_{N}=\alpha_{N}\left(\left(1_{U}\right)^{G}\right)_{N}=|G: U| \alpha_{N}
$$


and it follows that every irreducible constituent of the Brauer character $\alpha^{G}$ lies over $\theta$, as claimed. Also, if $\beta \in \operatorname{IBr}(G)$ is an irreducible constituent of $\alpha^{G}$, then $\beta_{N}$ is contained in $|G: U| \alpha_{N}$, which is a sum of $U$-conjugates of $\theta$ (by Clifford's Theorem applied to $\alpha)$. Hence, it follows that $\beta_{N}$ is a sum of some $U$-conjugates of $\theta$. Now, if $g \in G$, we have that $\theta^{g}$ is also an irreducible constituent of $\beta_{N}$. Therefore, there is $u \in U$ such that $\theta^{g}=\theta^{u}$. Hence, $g u^{-1} \in T$, and we conclude that $T U=G$. Now, since $N$ is contained in the kernel of every irreducible constituent of $\left(1_{U}\right)^{G}$, it follows that $\phi\left(1_{U}\right)^{G} \in \operatorname{cf}\left(G^{0} \mid \theta\right)$ and $\psi\left(1_{U}\right)^{G} \in \Delta$. Now,

$$
\phi\left(1_{U}\right)^{G}+\psi\left(1_{U}\right)^{G}=(\phi+\psi)\left(1_{U}\right)^{G}=\hat{\alpha}\left(1_{U}\right)^{G}=\left(\hat{\alpha}_{U}\right)^{G}=\alpha^{G}
$$

and we conclude that $\psi\left(1_{U}\right)^{G}=0$. Then

$$
\phi_{U}\left(\left(1_{U}\right)^{G}\right)_{U}=\left(\phi_{U}+\psi_{U}\right)\left(\left(1_{U}\right)^{G}\right)_{U}=\alpha\left(\left(1_{U}\right)^{G}\right)_{U}
$$

Since $\left(\left(1_{U}\right)^{G}\right)_{U}$ is never zero, we conclude that $\phi_{U}=\alpha$, as desired.

If $N \triangleleft G, \theta \in \operatorname{Irr}(N)$ and $T=I_{G}(\theta)$, recall that $\xi \mapsto \xi^{G}$ defines a bijection

$$
\operatorname{cf}\left(T^{0} \mid \theta\right) \rightarrow \operatorname{cf}\left(G^{0} \mid \theta\right)
$$

(as easily follows from Theorem (8.9) of [4]).

Lemma 2.4. Suppose that $N \subseteq U \subseteq G$, where $N \triangleleft G$. Let $\theta \in \operatorname{IBr}(N)$ and suppose that $\alpha \in \operatorname{IBr}(U \mid \theta)$ is $G$-stable. Write $T=I_{G}(\theta)$ and let $\xi \in \operatorname{IBr}(T \cap U \mid \theta)$ be the Clifford correspondent of $\alpha$ over $\theta$. Then $\xi$ is T-stable.

Proof. By Lemma 2.3, we know that $G=T U$. Also, by Lemma 2.3, let $\hat{\alpha} \in \operatorname{cf}\left(G^{0} \mid \theta\right)$ be such that $\hat{\alpha}_{U}=\alpha$. Now, let $\hat{\xi} \in \operatorname{cf}\left(T^{0} \mid \theta\right)$ be such that $\hat{\xi}^{G}=\hat{\alpha}$. By Mackey, we have that

$$
\left(\hat{\xi}_{T \cap U}\right)^{T}=\left(\hat{\xi}^{G}\right)_{U}=\hat{\alpha}_{U}=\alpha .
$$

We have that $\hat{\xi}_{T \cap U} \in \operatorname{cf}\left((T \cap U)^{0} \mid \theta\right)$, and we conclude that

$$
\hat{\xi}_{T \cap U}=\xi
$$

by uniqueness. Hence, by Lemma 2.1 we have that $\xi$ is $T$-stable, as desired.

The key idea in our proof of Theorem A is to use 'modular character triples'. The reader is referred to Chapter 7 of [4] for their definition and main properties.

Theorem 2.5. Suppose that $(G, N, \theta)$ is a modular character triple with $N$ a $\pi$-group. Then there exists an isomorphic triple $\left(G^{*}, N^{*}, \theta^{*}\right)$, where $N^{*}$ is a $\pi$-group contained in $Z\left(G^{*}\right)$.

Proof. We argue as in Theorem (5.2) of $[\mathbf{3}]$. Let $\left(G^{*}, N^{*}, \theta^{*}\right)$ be any isomorphic triple where $\theta^{*}$ is linear, and factor $\theta^{*}=\alpha \beta$, where the order of $\alpha$ is a $\pi$-number and the order of $\beta$ is a $\pi^{\prime}$-number. Notice that both characters are $G$-invariant, by uniqueness. As in Theorem (5.2) of [3] (and using Lemma (8.26) of [4] and its previous comments), it 
suffices to show that $\beta$ extends to $G$. By Theorem (8.29) of [4], it suffices to prove that $\theta$ extends to $Q^{*}$, where $Q^{*} / N^{*}$ is a Sylow $q$-subgroup of $G^{*} / N^{*}$. Suppose first that $q \in \pi$. Then the result follows from Theorem (8.23) of [4]. So we may assume that $q \in \pi^{\prime}$. Now, consider the group $Q$ corresponding to $Q^{*}$ with $N \subseteq Q \subseteq G$. Since $N$ is a $\pi$-group, $\theta$ extends to some $\psi \in \operatorname{IBr}(Q \mid \theta)$ by Theorem (8.13) of [4]. Let $\lambda=\psi^{*} \in \operatorname{Irr}\left(Q^{*} \mid \theta^{*}\right)$. Now, $\lambda$ is linear (because it extends $\theta^{*}$ ). Factor $\lambda$ as $\mu \nu$ with $o(\mu)$ a $\pi$-number and $o(\nu)$ a $\pi^{\prime}$-number. Then

$$
\mu_{N^{*}} \nu_{N^{*}}=\lambda_{N^{*}}=\theta^{*}=\alpha \beta,
$$

and, by uniqueness, we have that $\mu_{N^{*}}=\beta$, as desired.

Now we can prove Theorem A, which we restate here.

Theorem 2.6. Suppose that $G$ is $\pi$-separable and let $H$ be a Hall $\pi$-subgroup of $G$. If $\alpha \in \operatorname{IBr}(H)$ is $G$-stable, then $\alpha$ extends to some Brauer character of $G$.

Proof. We argue by double induction, first on $\left|G: \boldsymbol{O}_{\pi}(G)\right|$ and second on $|G|$. Let $N=\boldsymbol{O}_{\pi}(G)$ and let $\theta \in \operatorname{IBr}(N)$ be an irreducible constituent of $\alpha_{N}$. Let $T$ be the stabilizer of $\theta$ in $G$. We know that $T H=G$ by Lemma 2.3. Let $\beta \in \operatorname{IBr}(T \cap H \mid \theta)$ be the Clifford correspondent of $\alpha$ with respect to $\theta$. By Lemma 2.4, we know that $\beta$ is $T$-stable. Assume first that $T<G$. Then $\left|T: \boldsymbol{O}_{\pi}(T)\right|<|G: N|$, and by induction we have that $\beta$ extends to some $\xi \in \operatorname{IBr}(T)$. Now, by Mackey, we have that

$$
\left(\xi^{G}\right)_{H}=\left(\xi_{T \cap H}\right)^{H}=\beta^{H}=\alpha,
$$

as desired. So we may assume that $T=G$. Now, by Theorem 2.5 , let $\left(G^{*}, N^{*}, \theta^{*}\right)$ be an isomorphic modular character triple with $N^{*}$ a central $\pi$-group. Since $G / N$ and $G^{*} / N^{*}$ are isomorphic, we have in fact that $N^{*}=\boldsymbol{O}_{\pi}\left(G^{*}\right)$. Let $M=\boldsymbol{O}_{\pi^{\prime}}\left(G^{*}\right)$. If $M=1$, then $G^{*}=C_{G^{*}}\left(N^{*}\right) \subseteq N^{*}$, and in this case we are clearly done. So we may assume that $M>1$. Now, we work in $G^{*} / M$. Let $N^{*} \subseteq H^{*} \subseteq G^{*}$ correspond to $H$. Of course, notice that $H^{*}$ is a Hall subgroup of $G^{*}$. Suppose that $\alpha^{*} \in \operatorname{IBr}\left(H^{*}\right)$ corresponds to $\alpha$. By Lemmas 2.3 and 2.1, we know that $\alpha$ extends to some $\xi \in \operatorname{cf}\left(G^{0} \mid \theta\right)$. By the properties of modular character triples, we have that $\xi^{*}$ extends $\alpha^{*}$. Therefore, we have that $\alpha^{*}$ is $G^{*}$-stable. Since $H^{*} \cap M=1$, it follows that $\alpha^{*}$ uniquely extends to some Brauer character $\bar{\alpha} \in \operatorname{IBr}\left(H^{*} M\right)$ that contains $M$ in its kernel. View $\bar{\alpha}$ as an irreducible Brauer character of $H^{*} M / M$ and notice that $\bar{\alpha}$ is $G^{*} / M$-stable. Now, $\left|G^{*} / M: \boldsymbol{O}_{\pi}\left(G^{*} / M\right)\right|<|G: N|$, and by induction we have that $\bar{\alpha}$ extends to some Brauer character $\mu$ of $G^{*}$. Hence, $\alpha^{*}$ extends to $G^{*}$. Since $\mu$ necessarily lies over $\theta^{*}$, it follows that $\alpha$ extends to some Brauer character of $G$.

Next is Corollary B of $\S 1$.

Corollary 2.7. Suppose that $G$ is $\pi$-separable and let $H$ be a Hall $\pi$-subgroup of $G$. Then every irreducible Brauer character of $H$ extends to some Brauer character of $G$ if and only if whenever $x, y \in H$ are p-regular and $G$-conjugate, then $x$ and $y$ are $H$-conjugate. 
Proof. If $H$ controls $G$-fusion on $p$-regular elements, it is clear that every irreducible Brauer character of $H$ is $G$-stable. In this case, by Theorem A, every irreducible Brauer character of $H$ extends to some Brauer character of $G$. On the other hand, assume that every irreducible Brauer character of $H$ extends to some Brauer character of $G$. Then every irreducible Brauer character of $H$ is $G$-stable. Suppose now that $x, y \in H$ are $p$-regular and $G$-conjugate. Then $\alpha(x)=\alpha(y)$ for every $\alpha \in \operatorname{IBr}(H)$. In this case, $x$ and $y$ are $H$-conjugate, since the Brauer character table of $H$ is an invertible matrix (and, therefore, cannot have two identical columns).

Acknowledgements. Research partly supported by DGICYT.

\section{References}

1. P. X. Gallagher, Group characters and normal Hall subgroups, Nagoya Math. J. 21 (1962), 223-230.

2. I. M. IsAaCS, Induction and restriction of $\pi$-special characters, Can. J. Math. 38 (1986), 576-604.

3. I. M. IsaACS, Partial characters of $\pi$-separable groups, Progr. Math. 95 (1991), 273-287.

4. G. NAvarro, Characters and blocks of finite groups (Cambridge University Press, 1998). 